\section{Fight periodontitis with secure cloud-based}

Dentists can save time and fight back against periodontitis - one of the most widespread oral health complications in the world but seldom straightforward to assess for individual cases - with the help of PROPACS from PRO Diagnostics UK.

An innovative, cloud-based storage system for patient image files, PROPACS allows professionals to send radiographs to a team of radiology specialists for expert reporting.

Their special PerioGuide report is particularly useful as it not only indicates the severity of periodontal disease through an easy-to-understand, traffic-light-colour system, but it also gives detailed information on the case to help aid the formulation of an effective care plan.

The PerioGuide report reveals the amount of bone loss at each site of periodontal infection thus helping professionals to establish that the disease is present and also see how badly the patient is afflicted.

This saves dentists having to take these measurements themselves and the information can be sent straight to the hygienist and the periodontist, streamlining the whole process and providing better diagnostic confidence.

Dentists can also rest assured that their file storage system has absolute security. Encrypted with a state-of-the-art blend of safeguarding software, the PROPACS system is also GDPR compliant and easy to use.

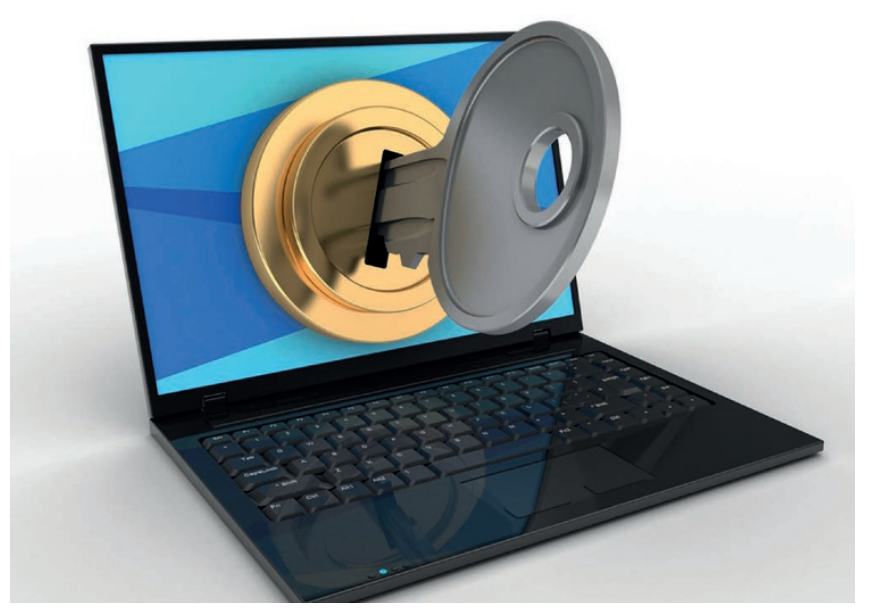

Furthermore, PROPACS helps to support dental professionals who use the system with continued education.

Members receive access to regular, complementary CPD courses that will help them keep up to date with the latest research and clinical education. Radiology specialists who have extensive knowledge in the field author each of the webinars and seminars, and these can be taken at any time so that users can benefit from training on their own terms.

More information is available at www.prodentalradiology.com or by emailing sales@prodiagnostics.co.uk.

\title{
Hygiene and therapy conference
}

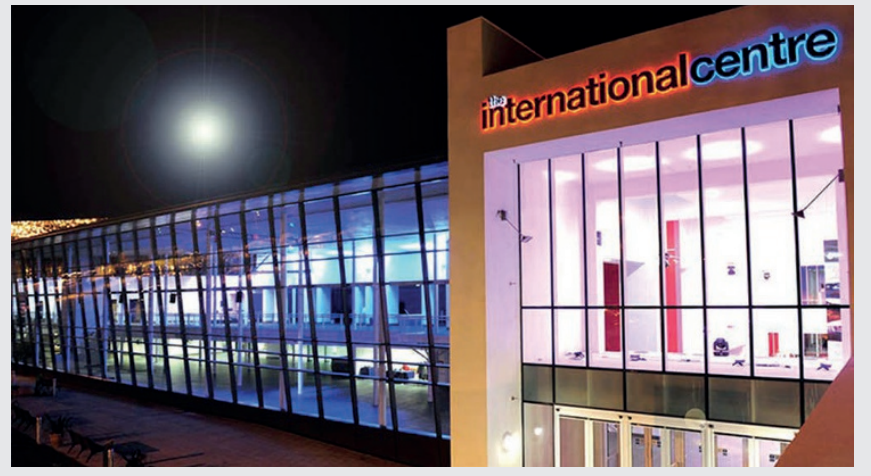

Built upon delegate feedback from previous events, the Oral Health Conference (OHC) 2018 being held on 23-24 November 2018 at Telford International Centre promises to set the stage for a bright future in dental hygiene and therapy.
During the flagship event of the British Society of Dental Hygiene and Therapy (BSDHT), this year's show will offer up to 12 hours of enhanced CPD covering a wide spectrum of relevant topics.

An exciting line-up of world-class speakers will impart knowledge and share expertise to help delegates refresh and refine their skills for the benefit of their patients.

The Annual Poster Competition (sponsored by Waterpik) will also provide an opportunity for professionals to showcase their own research and studies and gain recognition for their commitment from the wider dental community.

Open to dental hygienists, dental therapists and students, OHC 2018 is entitled 'The future is yours.

Early bird registration is open until 10 September for the very best price.

More information about the BSDHT is available at www.bsdht.uk or by calling 01788575050 or emailing enquiries@bsdht.org.uk.

\section{Goodwill survey now available}

A goodwill and benchmarking survey is now available directly from Dental Elite.

Since its mid-year goodwill report was released, Dental Elite has been monitoring a number of evolving trends, most notably the growing interest from private equity buyers who feel that there is money to be made in acquiring practices in a currently fragmented market.

The agency has also noted a considerable movement in the lower consolidator market as a result of a number of smaller mass operators, including Midland Smile Centres in the West Midlands and Avsan
Holdings, having cashed in their chips in the last year.

These findings have now been published in the new goodwill and benchmarking survey.

To find out more about the current state of the market - including growing regional differences in Multiple of Adjusted Net Profit and typical sale prices over the last financial year - or for help with buying or selling a practice, practitioners can contact Dental Elite.

For more information on Dental Elite visit www.dentalelite.co.uk or email info@dentalelite.co.uk or call 01788545900. 